\title{
Planar magnetoinductive wave transducers: Theory and applications
}

M. J. Freire, R. Marqués, F. Medina, M. A. G. Laso, and F. Martín

Citation: Appl. Phys. Lett. 85, 4439 (2004); doi: 10.1063/1.1814428

View online: https://doi.org/10.1063/1.1814428

View Table of Contents: http://aip.scitation.org/toc/apl/85/19

Published by the American Institute of Physics

\section{Articles you may be interested in}

Magnetoinductive waves in one, two, and three dimensions

Journal of Applied Physics 92, 6252 (2002); 10.1063/1.1510945

Planar magnetoinductive lens for three-dimensional subwavelength imaging

Applied Physics Letters 86, 182505 (2005); 10.1063/1.1922074

Electric-field-coupled resonators for negative permittivity metamaterials

Applied Physics Letters 88, 041109 (2006); 10.1063/1.2166681

A theory of metamaterials based on periodically loaded transmission lines: Interaction between magnetoinductive and electromagnetic waves

Journal of Applied Physics 97, 064909 (2005); 10.1063/1.1850182

Phonon-like dispersion curves of magnetoinductive waves

Applied Physics Letters 87, 072501 (2005); 10.1063/1.2011789

Electroinductive waves in chains of complementary metamaterial elements

Applied Physics Letters 88, 083503 (2006); 10.1063/1.2176850
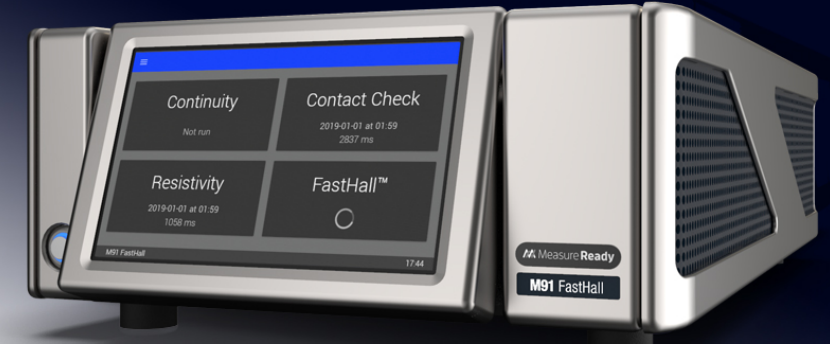

\section{M Measure Ready M91 FastHall ${ }^{\text {TM }}$ Controller}

A revolutionary new instrument for complete Hall analysis 


\title{
Planar magnetoinductive wave transducers: Theory and applications
}

\author{
M. J. Freire, ${ }^{a)}$ R. Marqués, and F. Medina \\ Departamento de Electrónica y Electromagnetismo, Facultad de Física, Universidad de Sevilla, \\ Avda. Reina Mercedes s/n, E 41012 Seville, Spain \\ M. A. G. Laso \\ Departmento de Ingeniería Eléctrica y Electrónica, Universidad Pública de Navarra, \\ E 31006 Pamplona, Navarra, Spain \\ F. Martín \\ Departament d'Enginyeria Electrónica, Universitat Autònoma de Barcelona, 08193 Bellaterra, \\ Barcelona, Spain
}

(Received 6 July 2004; accepted 17 September 2004)

\begin{abstract}
Transduction of magnetoinductive waves (MIWs) in planar technology is demonstrated. A transducer consisting of a one-dimensional periodic array of metallic split squared ring resonators (SSRR), placed between a pair of microstrip lines on a planar substrate has been fabricated and measured. The microstrip lines are inductively coupled to the SSRRs located at the ends of the periodic array and excite MIWs that propagate along the array. The theoretical model for the dispersion of MIWs is used to predict the dispersion relation and the delay time in the device. The delay time was measured and a good agreement was found with the theoretical predictions. The transmission coefficient of the device was also measured. The theoretical and experimental results suggest that the proposed configuration can find application in the design of delay lines and other microwave devices. In fact, the behavior of the proposed transducer is similar to that of the conventional ferrite magnetostatic-wave transducer. However, ferrite devices are fragile, difficult to integrate, and require a heavy external magnet or electromagnet to magnetize the ferrite to saturation. Since all these drawbacks are not present in the proposed configuration, it may be a useful alternative to those devices for many practical applications. () 2004 American Institute of Physics. [DOI: 10.1063/1.1814428]
\end{abstract}

Magnetoinductive waves (MIWs) ${ }^{1-4}$ are a new kind of guided electromagnetic (EM) waves conceived while investigating the EM properties of left-handed materials or metamaterials. ${ }^{5,6}$ These metamaterials simultaneously exhibit negative permeability and permittivity and can be fabricated by means of periodic arrays of electrically small magnetic resonators and wires. MIWs can propagate along a periodic array of these magnetic resonators due to the electromotive forces induced in the resonators by the magnetic coupling between them. In fact, MIWs are expected to be present in whatever periodic structure where the elements are magnetically coupled. The first magnetoinductive (MI) waveguide ${ }^{2}$ consisted of a one-dimensional array of capacitively loaded metallic loops arranged along the axis of a dielectric rod. This MI waveguide was fabricated and measured $^{2}$ to check experimentally the properties of the relation of dispersion ${ }^{1}$ of MIWs. Waveguide components such as junctions and directional couplers based on this MI waveguide have been proposed and theoretically analyzed. ${ }^{4}$

Since many of the present microwave devices are fabricated in planar technology it is of interest to study the transduction-i.e., the excitation and reception-of MIWs in this technology. In this letter, a planar MIW transducer is studied both theoretically and experimentally. The device is designed in order to allow the excitation, propagation, and detection of MIWs along a conventional planar circuit board in microstrip technology. MIW propagation along the device is shown by comparing the experimental results with those

\footnotetext{
${ }^{\text {a)} E l e c t r o n i c ~ m a i l: ~ f r e i r e @ u s . e s ~}$
}

provided by the MIW theory. MIWs are slow waves which can propagate only within a certain passband. ${ }^{1}$ This behavior recalls that of the magnetostatic waves (MSWs) induced in ferrimagnetic slabs magnetized to saturation. ${ }^{7,8}$ Therefore, it will be worth to compare the behaviors of the proposed planar MIW transducers and that of the well-known planar MSW transducers. ${ }^{8}$ MSW transducers are used for the design of delay lines and other microwave devices working in a similar range of frequencies. Some key characteristics of these devices, such as the group delay and the transmission coefficient, will be compared with those of the proposed MIW transducer. From this comparison, several advantages of the proposed configuration will come out.

Figure 1 shows both a photograph and a sketch of the proposed transducer. It consists of a pair of conventional input and output microstrip lines coupled to an array of resonant elements along which the MIWs propagate. Figure 2 shows in detail the layout of the resonant elements used for the periodic array. This resonant element consists of a deformation of the conventional split ring resonator geometry, ${ }^{9}$ which is intended to strength the coupling between adjacent elements. In the following we will call this configuration the split squared ring resonator (SSRR). The caption of Fig. 2 indicates the actual dimensions of the resonator in our experiment. The microstrip MIW transducer was fabricated on a planar circuit board of the type Arlon AR1000L-025-11, with dielectric constant $\varepsilon_{r}=10$ and thickness of $0.635 \mathrm{~mm}$ and with a metallic ground plane on the back side. On the upper side of the substrate (see Fig. 1), a set of five SSRRs and two microstrip lines were photoetched. The SSRRs 

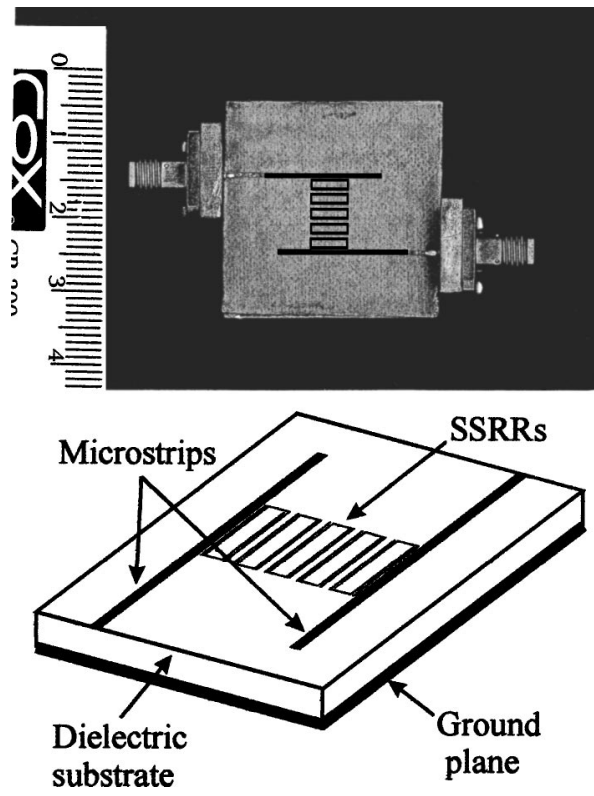

FIG. 1. Photograph and sketch of the planar MIW transducer. The dielectric substrate is supported on an aluminum mounting. Two coaxial connectors are soldered to the microstrip lines to facilitate the measurement with a vector network analyzer. The total length of the array is $1 \mathrm{~cm}$.

placed between the two microstrip lines constitute a finitelength planar MI waveguide. The first and the last SSRRs of this waveguide should be inductively coupled to the two microstrip lines for effective MIWs transduction. For this reason, the distance between the open end of both microstrip lines and the point of the line coincident with the center of the closest SSRR is a quarter wavelength at the resonant frequency of the SSRRs. Thus, the amplitude of the current is a maximum at this point, as well as the coupling between the lines and the SSRRs.

The dispersion relation of the MIWs is well known. ${ }^{1}$ Neglecting ohmic losses, it can be written as follows:

$$
\frac{\omega_{0}^{2}}{\omega^{2}}=1+\sum_{j=1}^{N} 2 \frac{M_{j}}{L} \cos (k j a), j=1,2, \ldots, N,
$$

where $\omega$ is the angular frequency, $\omega_{0}$ and $L$ are, respectively, the resonant frequency and the inductance of the resonant elements, $k$ is the wave number, $a$ is the periodicity of the array, and $M_{j}$ is the mutual inductance between two elements separated by a distance $j a$. The upper limit of summation $N$ in Eq. (1) has to be taken in order to provide a good convergence of the series and it depends on the coupling between elements ( $N$ increases as the coupling increases). In the MI waveguide analyzed by Shamonina et al. ${ }^{2}$ the line connecting the centers of the resonating loops is perpendicular to the plane of the loops. With this axial configuration, the interac-

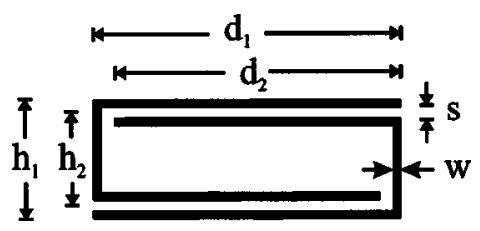

FIG. 2. SSRR drawn to scale $d_{2}=d_{1}-s-w$ is the overlaped length between the long arms. $h_{2}=h_{1}-s-2 w$ is the mean distance between long strips. Dimensions of the real SSRR in the experiment: $d_{1}=5 \mathrm{~mm}, h_{1}=1.47 \mathrm{~mm}, s$ $=0.127 \mathrm{~mm}, w=0.076 \mathrm{~mm}$. The periodicity of the array is $a=1.99 \mathrm{~mm}$. tion between far neighbors should be taken into account in the dispersion relation. In the planar configuration under study in the present work, even if the SSRRs are very close, it is a good hypothesis to assume only interaction between first neighbors. Therefore, in our case the dispersion relation (1) can be approximated to

$$
\frac{\omega_{0}^{2}}{\omega^{2}}=1+\frac{2 M}{L} \cos (k a),
$$

where $M$ is the mutual inductance between adjacent neighbors. The group delay defined as $\tau=-\delta \kappa / \delta \omega$ can be directly obtained from the above expression as

$$
\tau=-\frac{L}{M} \frac{\left(1+2 \frac{M}{L} \cos (k a)\right)^{3 / 2}}{\omega_{0} a \sin (k a)} .
$$

The minus sign in Eq. (3) and in the definition of $\tau$ is introduced in order to get a positive delay time since the mutual inductance $M$ is negative and wave propagation is backward in planar configurations. ${ }^{1}$ It has been mentioned above that the planar MIW transducer can find application as a microwave delay line. The delay time required by a microwave signal to travel along the periodic array of SSRRs in the device would be simply given by the product of the above-mentioned group delay $\tau$ and the length of the array (in our experiment this length is $1 \mathrm{~cm}$ as indicated in the caption of Fig. 1).

The resonant frequency $\omega_{0}$ can be calculated by means of a direct extension of a model ${ }^{10,11}$ which was previously proposed for the round split rings resonator configuration. This resonant frequency is given by

$$
\omega_{0}=\sqrt{\frac{2}{d_{2} L C_{\mathrm{pul}}}},
$$

where $C_{\mathrm{pul}}$ is the per unit length (pul) capacitance between the long arms of the SSRR and $d_{2}$ (see Fig. 2) is the overlapped length between the long arms. It should be noted that the two capacitors formed by the long arms are designed in such a way that the electric dipoles associated with them will cancel mutually when the SSRR is at resonance (note that this situation is just the opposite in conventional split rings resonators ${ }^{10}$ ). This avoids the existence of cross-polarization effects $^{10,11}$ and simplifies the analysis. The mutual inductance $M$ in Eqs. (2) and (3) and the total inductance $L$ are calculated by modeling the SSRR as two parallel wires of width $w$ and length $d_{1}$ (see Fig. 2) whose centers are separated by a distance $h_{2}\left(h_{2}\right.$ is the mean value between the outer and the inner width of the SSRR). These two wires should carry opposite currents in our model. The inductance of the lateral arms of length $h_{1}$ is neglected. In practical computations, both $C_{\text {pul }}$ and $L$ can be obtained from Bhartia's formulas ${ }^{12}$ for the characteristic impedance and the effective dielectric constant of a pair of coupled strips (these strips should have a width $w$ and should be separated by a distance $h_{2}$ for the calculation of $L$ and by a distance $s$ for the calculation of $C_{\text {pul }}$ ). Finally, the mutual inductance $M$ is calculated from elementary formulas of basic electromagnetism by neglecting the strip width $w$ in the aforementioned model.

Figure 3 shows the measured transmission coefficient in decibels versus the frequency in gigahertz in the transducer of Fig. 1. With the dimensions shown in the caption of Fig. 2 , the parameters of expression (3), deduced from the afore- 


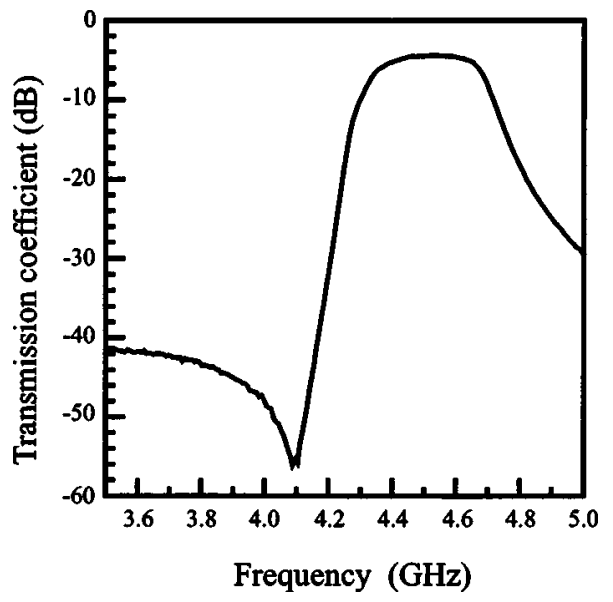

FIG. 3. Transmission coefficient in decibels vs frequency in gigahertz measured in the MIW transducer of Fig. 1.

mentioned models, are: $L=8.018 \mathrm{nH}, \quad M=-0.474 \mathrm{nH}, a$ $=1.99 \mathrm{~mm}$, and $f_{0}=\omega_{0} / 2 \pi=4.48 \mathrm{GHz}$. The measurements have been carried out with a vector network analyzer HP8510 B. The figure shows that there is a passband centered at the frequency of $4.48 \mathrm{GHz}$ predicted by our model. This seems to confirm that the signal transmitted between the input and the output microstrip lines is actually a MIW. The moderate insertion loss at the passband (around $3 \mathrm{~dB}$ ) suggests the usefulness of the proposed configuration for practical applications in microwave technology.

Figure 4 shows the delay time measured (solid line) in the transducer of Fig. 1. The theoretical delay time has been calculated by using expression (3) and has also been plotted (dashed lines). Figure 4 shows that the frequency range of the theoretical curve agrees with the frequency range where the experimental delay time is significant. This fact evidences the MIW propagation in the device. Moreover, the figure shows that there is only a difference of around $0.4 \mathrm{~ns}$ in the delay time between the predicted and the experimental values. This difference may be due to the fact that our model does not include the time required by the signal to travel along the two microstrip lines of the device. In fact, this time can be estimated from the total length of the microstrip lines and the group delay associated with the electromagnetic mode propagating along the microstrip lines. The group delay in the microstrip line can be calculated by using some of the commercial softwares available for the analysis of planar transmission lines. Thus, by using the commercial software TXLINE ${ }^{\mathrm{TM}}$, a group delay in the microstrip line of $0.09 \mathrm{~ns} / \mathrm{cm}$ is calculated at the resonant frequency of $4.48 \mathrm{GHz}$. Since

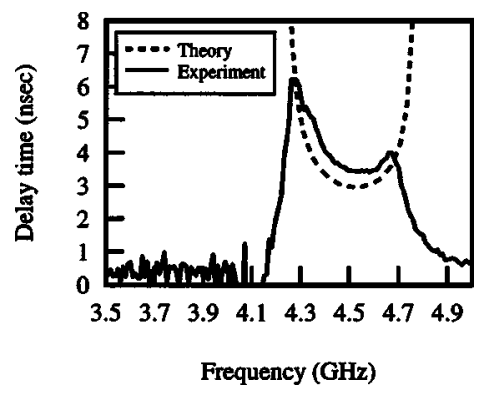

FIG. 4. Delay time in nanosecond vs frequency in gigahertz measured and calculated in the MIW transducer of Fig. 1. the two microstrip lines of the device sum a total length of $4.4 \mathrm{~cm}$, the group delay in the microstrip lines accounts for the major part of the difference in the curves of Fig. 4. However, a minor part of this difference may be also due to the interaction between far neighbors in the dispersion relation of MIWs, which has not been taken into account in the model.

The aforementioned results clearly suggest the usefulness of the proposed transducer for microwave filters, delay lines, and other microwave devices design. At the frequency range of operation of the device shown in Fig. 1, magnetostatic transducers are also of application. ${ }^{8}$ Therefore, a comparison between the performances of both designs is of interest. In fact, the reported magnitude of the transmission coefficient (Fig. 3) is of the same order as those present in typical low loss ferrite magnetostatic wave transducers. ${ }^{8}$ The reported delay time per centimeter (Fig. 3), although significant, is lower than the typical delay time in magnetostatic surface-wave transducers by an order of magnitude, and lower than the typical delay time in magnetostatic volumewave transducers by two orders of magnitude. Anyway, the reported delay time is still higher than the time taken by light in traveling the same distance by two orders of magnitude. In addition, it should be taken into account that the proposed device has only demonstration purposes, and has not been optimized at all. Moreover, magnetostatic wave transducers use ferrite slabs magnetized to saturation, which requires the presence of an external permanent magnet or electromagnet. Magnets are heavy and bulky, and ferrites are expensive and fragile and cannot be easily integrated. On the contrary, MIW transducers can be easily implemented in a printed circuit board by the same photoetching techniques used for manufacturing the whole circuit, and are robust and inexpensive. In addition, they do not require an external magnet or electromagnet. Therefore, MIW transducers seem to have some advantages over conventional magnetostatic wave transducers, which suggest future applications of those devices in microwave technology.

This work has been supported by DGI and CICYT by Project Contract Nos. BFM2001-2001, TIC2002-04528-C0201, and TIC2001-3163.

${ }^{1}$ E. Shamonina, V. A. Kalinin, K. H. Ringhofer, and L. Solymar, J. Appl. Phys. 92, 6252 (2002).

${ }^{2}$ E. Shamonina, V. A. Kalinin, K. H. Ringhofer, and L. Solymar, Electron. Lett. 38, 371 (2002).

${ }^{3}$ M. C. K. Wiltshire, E. Shamonina, I. R. Young, and L. Solymar, Electron. Lett. 39, 215 (2003).

${ }^{4}$ E. Shamonina and L. Solymar, J. Phys. D 37, 362 (2004).

${ }^{5}$ D. R. Smith and N. Kroll, Phys. Rev. Lett. 85, 2933 (2000).

${ }^{6}$ D. R. Smith, W. J. Padilla, D. C. Vier, S. C. Nemat-Nasser, and S. Schultz, Phys. Rev. Lett. 84, 4184 (2000).

${ }^{7}$ M. S. Sodha and N. C. Srivastava, Microwave Propagation in Ferrimagnetics (Plenum, New York, 1981).

${ }^{8}$ P. Kabos and V. S. Stalmachov, Magnetostatic Waves and their Applications (Chapman \& Hall, London, 1994).

${ }^{9}$ J. B. Pendry, A. J. Holden, D. J. Robbins, and W. J. Stewart, IEEE Trans. Microwave Theory Tech. 47, 2075 (1999).

${ }^{10}$ R. Marques, F. Medina, and R. Rafii-El-Idrissi, Phys. Rev. B 65, 144440 (2002).

${ }^{11}$ R. Marques, F. Mesa, J. Martel, and F. Medina, IEEE Trans. Antennas Propag. 51, 2572 (2003).

${ }^{12}$ P. Bhartia and I. Bahl, Microwave Solid State Circuit Design (Wiley, New York, 1988). 